\title{
Is Inflammation the Link between Atherosclerosis and Vascular Calcification in Chronic Kidney Disease?
}

\author{
George Tsirpanlis \\ Department of Nephrology, General Hospital of Athens, Athens, Greece
}

\author{
Key Words \\ Renal failure $\cdot$ Cardiovascular disease $\cdot$ Oxidized LDL . \\ Calcium $\cdot$ Phosphorus $\cdot$ Fetuin-A $\cdot$ RANKL . \\ Osteoprotegerin $\cdot$ Proinflammatory cytokines
}

\begin{abstract}
Atherosclerosis and vascular calcification often co-exist in chronic kidney disease (CKD) patients. Although the former has been recently recognized as an active inflammatory process, atherosclerosis-related calcification of the intima is still viewed as a passive epiphenomenon. Recent experimental data showed that ossification of the internal vascular wall might also be an active inflammatory process interrelated to atherosclerosis. Factors like RANKL (receptor activator of nuclear factor $\kappa \mathrm{B}$ ligand), RANK and osteoprotegerin modulate vascular calcification and at the same time are involved in the process of atherosclerosis. Moreover, basic calcium phosphate crystals could interact with and activate monocytesmacrophages that produce proinflammatory cytokines capable of initiating - via endothelial activation and leukocyte adhesion - the atherosclerotic process. Thus, vascular calcification might be an active player and not simply an epiphenomenon in atherosclerosis. Should the above-mentioned data be confirmed in future studies, calcification of the internal vascular wall and atherosclerosis might be viewed and treated as tightly interconnected and linked by inflammation processes in CKD patients.
\end{abstract}

Cardiovascular morbidity and mortality is known to be increased in patients with chronic kidney disease (CKD). Atherosclerosis, which is influenced by both traditional and non-traditional (e.g. oxidative stress, albuminuria, anemia, etc.) cardiovascular risk factors, and has recently been characterized as an inflammatory process, is considered to be the main cause of the elevated cardiovascular risk associated with CKD. The CKD population also often presents with vascular calcification. Atherosclerosis-related calcification of the intima appears to be of particular clinical importance, although the cardiac valves and medial arterial layer are also often involved [1]. Although ossification of blood vessel walls is no longer considered a passive process, it is still believed to follow, rather than actively participate in, the development of the atherosclerotic plaque. Recent data offer a novel perspective on the role of vascular calcification in atherosclerosis in patients with CKD.

Regulation of vascular calcification by the osteoclast regulatory factors RANKL (receptor activator of nuclear factor $\kappa \mathrm{B}$ ligand), its receptor RANK, and osteoprotegerin influences the inflammatory component of atherosclerosis [2]. The release of RANKL from endothelial cells is induced by proinflammatory cytokines (tumor necrosis factor (TNF), interleukin (IL)-1, etc.) or by adherence of activated lymphocytes to the vascular wall at the site of atherosclerosis. RANKL promotes osteoclastic transformation of monocytes and osteogenic differentiation of vascular smooth muscle cells (processes involved in vas-

\section{KARGER \\ Fax +41613061234 E-Mail karger@karger.ch} www.karger.com
George Tsirpanlis, MD

Amarysias Artemidos 34C

GR-15124 Marousi/Athens (Greece)

Tel./Fax +30 2106854393

E-Mail tsipg@ath.forthnet.gr 


\section{VASCULAR LUMEN}
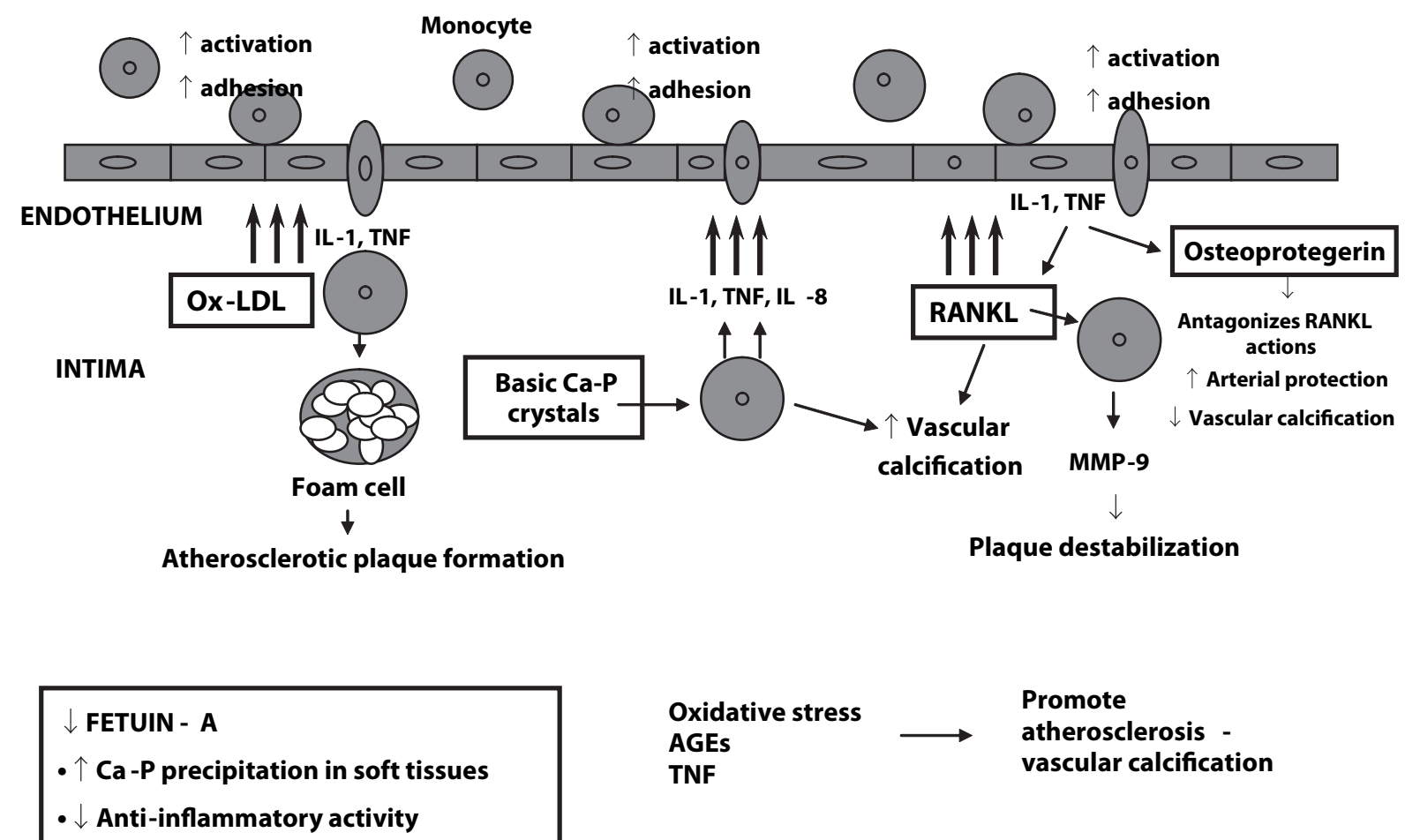

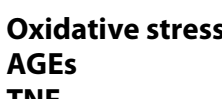

TNF
Promote atherosclerosis vascular calcification
Fig. 1. Involvement of factors modulating vascular calcification in the process of atherosclerosis via inflammation. Basic calcium phosphate (Ca-P) crystals and RANKL activate endothelium, increase adhesion and facilitate circulating monocytes' entry in vascular intima - the first step in the process of atherosclerosis in a manner similar to that of oxidized LDL (Ox-LDL) (left side of figure). Mediators are mainly proinflammatory cytokines (IL-
1, TNF, IL-8). Osteoprotegerin counterbalances RANKL effects. Fetuin, a negative acute phase protein frequently found to be low in CKD patients, except from vascular calcification antagonism, has also anti-inflammatory activities. Oxidative stress, AGEs, and TNF not only promote atherosclerosis but also, mostly via inflammation induction, vascular calcification. MMP-9 = Metalloproteinase-9; foam cell $=$ macrophages containing oxidized lipids . cular calcification), and it also induces endothelial chemotactic migration of monocytes to the endothelium and matrix metalloproteinase-9 activity in monocytes (processes essential for the development and destabilization of the atherosclerotic plaque $[3,4]$ ) (fig. 1). By contrast, osteoprotegerin has anti-inflammatory and anti-atherosclerotic properties, and it exerts the opposite effects to RANK signaling [2, 5] (fig. 1).

As mentioned above, atherosclerosis is a chronic inflammatory process $[3,6]$. To dampen inflammation, activated monocytes and macrophages must be deactivat- ed, and participating apoptotic cells must be cleared via phagocytosis [7]. The negative acute-phase reactant fetuin-A - an inhibitor of calcium-phosphorus precipitation in soft tissues - appears to contribute to the resolution of inflammation, by enhancing the above-mentioned processes $[8,9]$. Moreover, fetuin-A is believed to have antifibrotic activity $[10,11]$, and has been shown to inhibit apoptosis of vascular smooth muscle cells [3, 12], a process known to contribute to the destabilization of atherosclerotic plaque. Taking into consideration that hepatic synthesis of fetuin-A is expected to be suppressed in 
many CKD patients - due to chronic inflammatory state [13] - the above-described activities of this protein are also expected to be diminished.

Vascular calcification, as part of the atherosclerotic process, is due to the deposition in the arterial intima of basic calcium phosphate (BCP) crystals, consisting mainly of calcium hydroxyapatite $\left(\mathrm{Ca}_{10}\left(\mathrm{PO}_{4}\right)_{6}(\mathrm{OH})_{2}\right)$ and similar to those that mineralize bone. Recently, in a very interesting study, Nadra et al. [14] showed that BCP crystals can interact with and activate human monocyte-derived macrophages, inducing a proinflammatory state. Specifically, internalization of BCP microcrystals into the vacuoles of macrophages was associated with secretion of proinflammatory cytokines (including TNF, IL-1 and IL-8) capable of activating endothelial cells and promoting leukocyte adhesion [14] (fig. 1). Endothelial activation and leukocyte adhesion are the initial steps in atherosclerosis [3]. These new data 'place BCP crystal deposition at the very heart of atherosclerosis' [15]. If calcium phosphorus crystals act as antigens that activate the main cell types participating in the chronic inflammatory response - namely monocytes and macrophages - these crystals would have essentially the same stimulatory action as other well-known atherogenic factors such as oxidized LDL cholesterol (fig. 1). Thus, vascular calcification might be an active player and not simply an epiphenomenon in atherosclerosis. If so, inflammation would be the connecting link once more.

The vascular endothelium also reacts with oxidized LDL cholesterol, leading to increased expression of genes that affect vascular calcification (e.g., matrix gla protein) [16]. Factors related to inflammation and atherosclerosis, such as advanced glycation end products (AGEs) and oxidative stress, might also be involved in this process [17,
18]. AGEs induce osteoblastic differentiation of pericytes which are mesenchymal progenitors - and contribute to the development of vascular calcification in diabetes [17], while oxidative stress modulates osteoblastic differentiation of calcifying vascular cells too [18]. Furthermore, TNF promotes calcification of vascular cells in vitro [19]. All the above findings add weight to the theory that vascular calcification is actively related to the inflammatory process of atherosclerosis.

Atherosclerosis, inflammation and vascular calcification characterize CKD patients. Although atherosclerosis is caused by many traditional and non-traditional cardiovascular risk factors and vascular calcification is related to the abnormalities in calcium and phosphorus metabolism that are frequently observed in this population, inflammation could be the missing link connecting both [6]. Factors like RANKL, fetuin-A, proinflammatory cytokines and calcium-phosphorus crystals appear to actively contribute to both vascular calcification and atherosclerosis. As the above-mentioned data indicate, the high prevalence of accelerated atherosclerosis in CKD could be convincingly explained by the existence of an active interplay between vascular calcification and atherosclerosis via inflammation, against a background of severe calcium-phosphorus disturbances.

If the above-mentioned theory is further supported by forthcoming studies, then the treatment of all three entities (atherosclerosis, calcium-phosphorus disturbances and inflammation) have to be instituted early, concomitantly and intensively in these patients. Medications such as statins, angiotensin-converting enzyme inhibitors and sevelamer hydrochloride might be a good choice mainly due to their pleiotropic effects.

\section{References}

1 London GM, Guerin AP, Marchais SJ, Metivier F, Pannier B, Adda H: Arterial media calcification in end-stage renal disease: impact on all-cause and cardiovascular mortality. Nephrol Dial Transplant 2003;18:17311740 .

-2 Collin-Osdoby P: Regulation of vascular calcification by osteoclast regulatory factors RANKL and osteoprotegerin. Circ Res 2004; 95:1046-1057.

>3 Glass KC, Witztum JL: Atherosclerosis: the road ahead. Cell 2001;104:503-516.
$>$

Gough PJ, Gomez IG, Wille PT, Raines EW: Macrophage expression of active MMP-9 induces acute plaque disruption in apoE-deficient mice. J Clin Invest 2006;116:59-69.

5 Bennett BJ, Scatena M, Kirk EA, Rattazzi M, Varon RM, Averill M, Schwartz SM, Giachelli CM, Rosenfeld ME: Osteoprotegerin inactivation accelerates advanced atherosclerotic lesion progression and calcification in older $\mathrm{ApoE}^{-/-}$mice. Atherioscler Thromb Vasc Biol 2006;26:2117-2124.

-6 Tsirpanlis G: Inflammation in atherosclerosis and other conditions: a response to danger. Kidney Blood Press Res 2005;28:211217.
7 Henson PM: Dampening inflammation. Nat Immunol 2005;6:1179-1181.

$>8$ Wang $\mathrm{H}$, Zhang M, Bianchi M, Sherry B, Sama A, Tracey KJ: Fetuin ( $\alpha_{2}$-HS-glycoprotein) opsonizes cationic macrophage-deactivating molecules. Proc Natl Acad Sci USA 1998;95:14429-14434.

$\checkmark 9$ Jersmann HP, Dransfield I, Hart SP: Fetuin/ $\alpha_{2}$-HS glycoprotein enhances phagocytosis of apoptotic cells and macropinocytosis by human macrophages. Clin Sci (Lond) 2003; 105:273-278. 
10 Demetriou M, Binkert C, Sukhu B, Tenenbaum HC, Dennis JW: Fetuin $/ \alpha_{2}-\mathrm{HS}$ glycoprotein is a transforming growth factor- $\beta$ type II receptor mimic and cytokine antagonist. J Biol Chem 1996;22:12755-12761.

- 11 Merx MW, Schafer C, Westenfeld R, Brandenburg V, Hidajat S, Weber C, Ketteler M, Jahnen-Dechent W: Myocardial stiffness, cardiac remodeling, and diastolic dysfunction in calcification-prone fetuin-A-deficient mice. J Am Soc Nephrol 2005; 16:33573364.

-12 Reynolds JL, Skepper JN, McNair R, Kasama T, Gupta K, Weissberg PL, Jahnen-Dechent W, Shanahan CM: Multifunctional roles for serum protein fetuin-A in inhibition of human vascular smooth muscle cell calcification. J Am Soc Nephrol 2005;16:2920-2930.
13 Tsirpanlis G: The pattern of inflammation and a potential new clinical meaning and usefulness of C-reactive protein in end-stage renal failure patients. Kidney Blood Press Res 2005;28:55-61.

14 Nadra I, Mason JM, Philippidis P, Florey O, Smythe C, McCarthy GM, Landis RC, Haskard DO: Proinflammatory activation of macrophages by basic calcium phosphate crystals via protein kinase $\mathrm{C}$ and MAP kinase pathways: a vicious cycle of inflammation and arterial calcification? Circ Res 2005; 96:1248-1256.

15 Bostrom K: Proinflammatory vascular calcification. Circ Res 2005;96:1219-1220.
16 Cola C, Almeida M, Li D, Romeo F, Metha JL: Regulatory role of endothelium in the expression of genes affecting arterial calcification. Biochem Biophys Res Commun 2004; 320:424-427.

17 Yamagishi S, Fujimori H, Yonekura H, Tanaka N, Yamamoto $\mathrm{H}$ : Advanced glycation end-products accelerate calcification in microvascular pericytes. Biochem Biophys Res Commun 1999;258:353-357.

18 Mody N, Parhami F, Sarafian TA, Demer LL: Oxidative stress modulates osteoblastic differentiation of vascular and bone cells. Free Radic Biol Med 2001;31:509-519.

19 Tintut Y, Patel J, Parhami F, Demer LL: Tumor necrosis factor- $\alpha$ promotes in vitro calcification of vascular cells via the cAMP pathway. Circulation 2000;102:2636-2642. 